\title{
In GaN Multiple Quantum Well Solar Cells on a Patterned Sapphire Substrate
}

\author{
Zhen $\mathrm{Bi}^{1,2,3}$ \\ ${ }^{1}$ School of Physics and Optoelectronic Engineering, \\ Xidian University, Xi'an, 710071, China \\ ${ }^{2}$ School of Advanced Material and Nanotechnology, \\ Xidian University, Xi'an, 710071, China \\ ${ }^{3}$ The State Key Discipline Laboratory of Wide Band Gap \\ Semiconductor Technology, \\ Xidian University, Xi'an, 710071, China.
}

\author{
Jincheng Zhang ${ }^{1}$, Yue Hao ${ }^{1}$ \\ ${ }^{1}$ School of Physics and Optoelectronic Engineering, \\ Xidian University, \\ Xi'an, 710071, China
}

\begin{abstract}
The performance In GaN/GaN multiple quantum wells solar cells on a patterned sapphire substrate (PSS) was investigated. Compare to the solar on a planar substrate, the In GaN/GaN multiple quantum wells (MQWs) solar cell grown on PSS exhibited a higher performance, with the conversion efficiency $(\eta)$ rising from $0.36 \%$ to $0.64 \%$.
\end{abstract}

Keywords-In GaN; multiple quantum wells; solar cells; conversion efficiency

\section{INTRODUCTION}

As a tunable bandgap material with predicted radiation resistance among the III-Nitride materials, InGaN has attracted much attention in the field of space solar cells in the past years $[1,2]$. This can be largely attributed to its wide and continuous bandgap coverage $(0.69-3.4 \mathrm{eV})$ in solar spectrum, just by changing indium content in the InGaN alloy [3, 4].

On the other hand, there are several challenges to the device designer to take full advantage of III-N materials' potential $[5,6]$. First, the lattice mismatch between InGaN and $\mathrm{GaN}$ will induce the strain relaxation in growth and results in high dislocation density, which will degrade the crystal quality of the films and lower the device performance. Second, spontaneous and piezoelectric polarization will also introduce charges at the interface of InGaN/GaN, which may be detrimental to obtaining a high conversion efficiency solar cell. In addition, with the increase of In content and the thickness, the InGaN layer will generate more carriers which will be beneficial to improving conversion efficiency. However, it is difficult to obtain the high quality InGaN layer with both high thickness and In content.

As mentioned above, on account of a compromise between In content and film thickness, researchers have to choose multiple quantum wells (MQWs) structure as light absorption layer [7]. Although main efforts have been focused in the InGaN/GaN MQWs solar cells so far, the device performance is still not improved significantly. In this paper, we reported on the performance of InGaN/GaN MQWs solar cells on two different sapphire substrates.

\section{EXPERIMENT}

As is illustrated in Figure 1, the two kinds of device structures were same except for the different substrates. One was a patterned sapphire substrate (PSS), and the other was a common planar substrate. Both of the two samples were grown on $2 \mu \mathrm{m}$ thick GaN: Si templates by metal-organic chemical-vapour deposition (MOCVD). The InGaN/GaN MQWs of the two samples both had 5 periods, and the thickness of $\mathrm{InGaN}$ wells and $\mathrm{GaN}$ barriers were about $3.5 \mathrm{~nm}$ and $9.5 \mathrm{~nm}$, separately. In composition in the InGaN well was estimated about $12 \%$ by X-Ray Diffraction (XRD). The XRD of the samples were measured by Bruker D8 Discover HRXRD using the

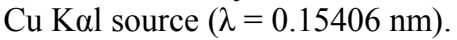

Before device fabricating process, the Indium Tin Oxide (ITO) conductive expanding layer was deposited by electron beam evaporation at first. The device was then fabricated into $1 \mathrm{~mm} \times 1 \mathrm{~mm}$ mesas by standard contact lithography and inductively coupled plasma etching. Finally, Ohmic contacts to p-GaN and n-GaN were both deposited by electron beam evaporation of $\mathrm{Cr} / \mathrm{Ni} / \mathrm{Au}$ and then rapidly thermal annealed at $300^{\circ} \mathrm{C}$ in $\mathrm{N}_{2}$ for $10 \mathrm{~min}$. The grid $\mathrm{p}-\mathrm{GaN}$ electrodes on top of the mesa were $5 \mu \mathrm{m}$ wide and $65 \mu \mathrm{m}$ center to center grid spacing.

The device current-voltage characteristic was measured by a HP 4156 instrument. The device was illuminated by a SAN-EI solar simulator with a Xe lamp and an AM1.5G filter. The illumination spectrum was calibrated by a Si standard solar cell. 


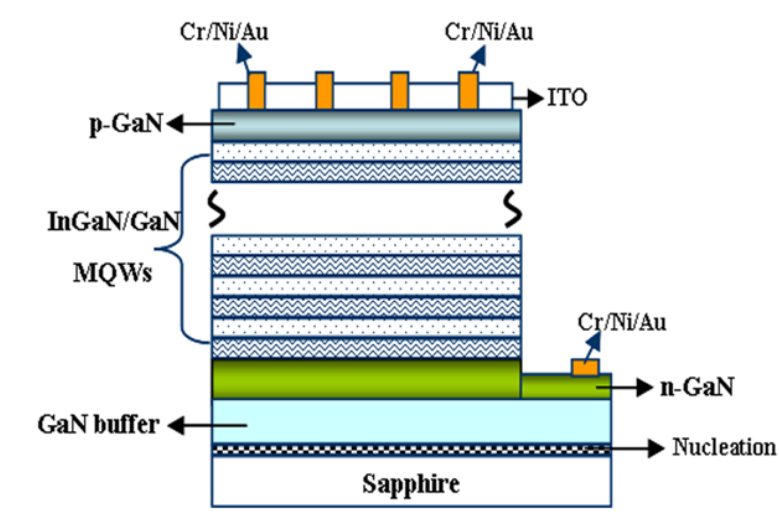

Figure 1. The structure of InGaN/GaN MQWs solar cell

\section{MEASUREMENT AND DISCUSSION}

Figure 2 (a) shows the XRD $\omega-2 \theta$ scans of the samples separately grown on PSS and planar substrates. The In content in the InGaN well for both solar cells is estimated at $12 \%$ by the fitting curve. The thickness of GaN barrier and $\mathrm{In}_{0.12} \mathrm{Ga}_{0.88} \mathrm{~N}$ well are estimated at $9.5 \mathrm{~nm}$ and $3.5 \mathrm{~nm}$, according to the spacing of the adjacent diffraction peaks [8]. Figure 2 (b) shows the (002) $\omega$ rocking curves of two samples. The FWHM are 207 and 256 arcseconds, respectively, which indicates that the film grown on PSS has higher crystal quality and lower dislocation density [9, 10]. The growth on the PSS is beneficial to releasing the strain due to the mismatch between $\mathrm{GaN}$ and sapphire substrate and decreasing the dislocations by lateral growth. It has been reported in others' works that there are a large number of stacking faults appeared during the initial growth of GaN buffer layer. These stacking faults interact with the threading dislocation (TD) defects, preventing further penetration into the MQWs [11].

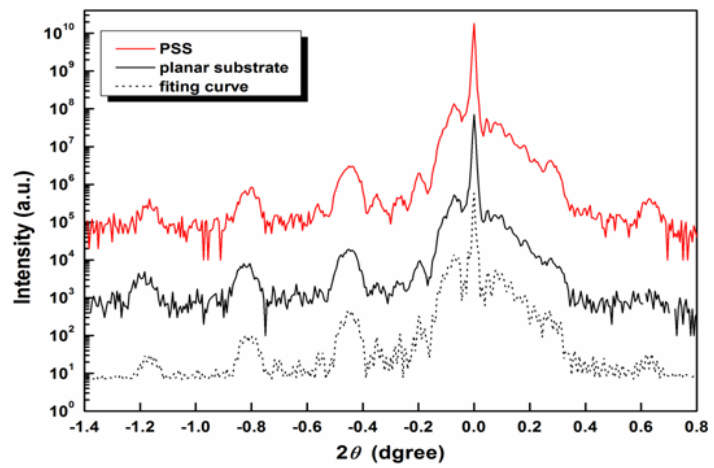

(a) $\omega-2 \theta$ scans

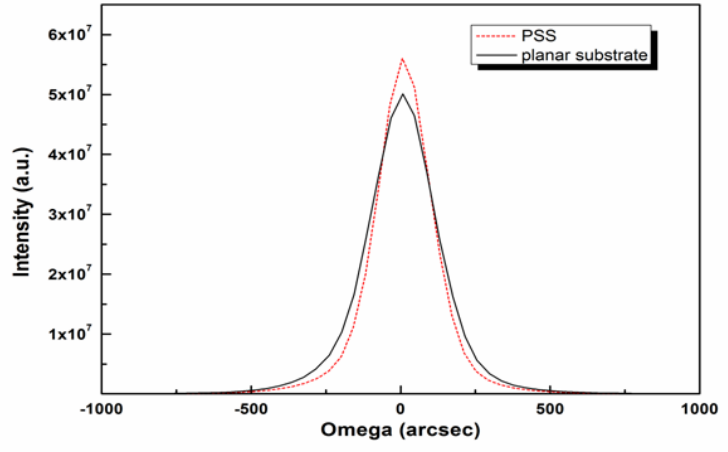

(b) $\omega$ rocking curves

Figure 2. XRD results of the films on PSS and planar substrates

Figure 3 shows the comparison of $\mathrm{J}-\mathrm{V}$ characters of these two samples. It is clear that the performance of the devices on PSS is better than the other one on the planar substrate. The reason is that the film grown on PSS exhibit better crystal qualities and optical qualities, which will be beneficial to enhancing light absorption and increasing the photon-generated carriers [11]. At the same time, it is also helpful to extending the light path length in the device and absorbing more photons. Therefore, the Jsc and Voc of the sample on PSS are both comparably higher than the one on planar substrate. Relatively, the conversion efficiency also rises from $0.36 \%$ to $0.64 \%$.

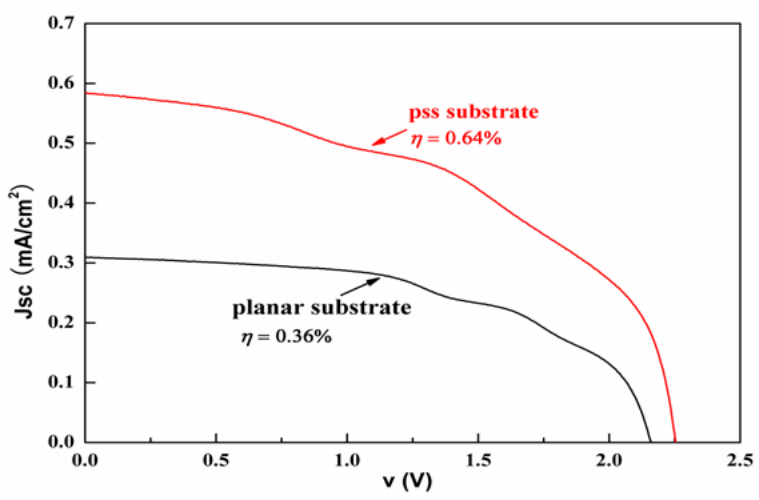

Figure 3. J-V characteristics of InGaN solar cells at different substrates.

\section{CONCLUSIONS}

In summary, the performances of InGaN/GaN MWQs solar cells grown on two different substrates were investigated. The samples on PSS exhibited a higher performance than that on planar substrates. Compared to the device on planar substrate, Jsc and Voc of the device on PSS are both higher. Therefore, the conversion efficiency also relatively rises from $0.36 \%$ to $0.64 \%$. 


\section{REFERENCES}

[1] Junqiao Wu, When Group III - Nitrides Go Infrared: New Properties and Perspectives, J. Appl. Phys., 106, 011101, 2009.

[2] Akio Yamamoto, Md. R. Islam, Ting-Ting Kang, and Akihiro Hashimato, Recent advances in InN-based solar cells: status and chanllenges in InGaN and InAIN solar cells, Phys. status solidi C7, No.5, pp. 1309-1316, 2010.

[3] J.J.Wierer.Jr., D.D.Koleske, and S.R.Lee, Influence of barrier thickness on the performance of InGaN/GaN multiple quantum well solar cells, Appl.Phys.Lett., 100, 111119, 2012.

[4] Carl J. Neufeld, Samantha C. Cruz, Robert M. Farrel, Michael Iza, Jordan R Lang,Staia Keller, Shuji Nakamura, Steven P. DenBaars, James S. Speck, and Umesh K. Mishra, Effect of doping and polarization on carrier collection in InGaN quantum well solar cells, Appl.Phys.Lett., 98, 243507, 2008.

[5] R.Dahal, B. Pantha, J. Li, J. Y. Lin, and H. X. jiang, InGaN/GaN multiple quantum well solar cells with long operating wavelengths, Appl.Phys.Lett., 94, 063505, 2009.

[6] K.Y. Lai, G. J. Lin, Y. L. Lai, Y. F. Chen, and J. H. He, Effect of indium fluctuation on the photovoltaic characteristics of InGaN/GaN multiple quantum well solar cells, Appl. Phys. Lett., 96, 081103(2010).
[7] R. Dahal, J. Li, K.Aryal, J. Y. Lin, and H.X. Jiang, InGaN/GaN multiple quantum well concentrator solar cells, Appl. Phys. Lett.,97, 073115,2010

[8] Chia-Lung Tsai, Gong-Chen Fan, and Yu-Sheng Lee, J. Vac. Sci. Technol. B 29(2), 021201, 2011.

[9] Z. H. Feng, Y. Qi, A. Lu, and K. Lau, GaN-based blue lightemitting diodes grown and fabricated on patterned sapphire substrates by metalorganic vapor-phase epitaxy, J. Cryst. Growth, 272, pp. 327-332, 2004.

[10] H. Cho, J. Lee, K. Kim, G. Yang, J. Song, and P. Yu, Effect of buffer layers and stacking faults on the reduction of threading dislocation density in $\mathrm{GaN}$ overlayers grown by metalorganic chemical vapor deposition, J. Appl. Phys., 89, 2617, 2001.

[11] Ya-Ju Lee, Min-Hung Lee, Chun-Mao Cheng. and Chia-Hao Yang, Enhanced conversion efficiency of InGaN multiple quantum well solar cells grown on a patterned sapphire substrate, Appl. Phys. Lett., 98, 263504, 2011.

[12] R. H. Horng, W. K. Wang, S.C. Huang, S. Y. Huang, S. H. Lin, C .F. Lin, and D. S. Wuu, Growth and characterization of $380 \mathrm{~nm}$ InGaN/AlGaN LEDs grown on patterned sapphire substrates, $J$. Cryst. Growth, 298, pp. 219-222, 2007. 